\title{
FEMORAL TUNNELS' LENGTH CHANGES WITH KNEE FLEXION ANGLE IN ANATOMICAL ANTERIOR CRUCIATE LIGAMENT RECONSTRUCTION
}

Julio Cesar Gali ${ }^{1}$, Heetor Campora de Sousa Oliveira², Bruno Asprino Ciâncio², Marcos Vianna Palma², Ricardo Kobayashi ${ }^{2}$, Edie Benedito Caetano ${ }^{3}$

\begin{abstract}
Objective: The objective of our study was to evaluate the effect that knee flexion angle while femoral tunnels are being drilled may have on the length of these tunnels, in anatomical reconstruction of the anterior cruciate ligament. Methods: We measured the lengths of anteromedial and posterolateral tunnels for the anterior cruciate ligament in 20 unpaired anatomical knee specimens (10 right and 10 left knees), all with the cartilage and cruciate ligaments intact. Tunnels were drilled with the knees flexed at $90^{\circ}, 110^{\circ}$ and $130^{\circ}$, through the accessory anteromedial portal, with a $2.5 \mathrm{~mm}$ drill. The statistical analysis was done by means of Friedman's variance analysis and the Mann-Whitney U test. Results: The mean anteromedial femoral tunnel lengths measured with the knees flexed
\end{abstract}

at $90^{\circ}, 110^{\circ}$ and $130^{\circ}$ were $33.7( \pm 3.72) \mathrm{mm}, 37.4( \pm 2.93)$ $\mathrm{mm}$ and $38.8( \pm 3.31) \mathrm{mm}$, respectively. For the posterolateral femoral tunnel lengths, the results were $32.1( \pm 4.24) \mathrm{mm}, 37.3$ $( \pm 4.85) \mathrm{mm}$ and $38.4( \pm 2.51) \mathrm{mm}$, respectively. Friedman's variance analysis showed that there was a significant difference between the lengths of the tunnels drilled with $90^{\circ}$ and $110^{\circ}$ of flexion angle, but showed that there was no significant difference between the tunnels drilled with flexion of $110^{\circ}$ and $130^{\circ}(\mathrm{P}<0.05)$. Conclusions: It is possible to drill the femoral tunnels through the accessory anteromedial tunnel with the knee flexed at $110^{\circ}$ in such a way as to produce a tunnel of sufficient length for a good bone-graft interface.

Keywords - Anterior Cruciate Ligament; Knee; Femur; Tibia; Reconstructive Surgical Procedures

\section{INTRODUCTION}

Today, there is a trend towards changes in the technique of surgical reconstruction of the anterior cruciate ligament (ACL). Until recently, it was recommended that in the arthroscopic reconstruction of this ligament, the femoral tunnel should be constructed through the tibial tunnel, which predisposes towards a higher location in the intercondyle for the femoral tunnel, in a non-anatomical position ${ }^{(1)}$.

The new tendency is to seek to reconstruct this ligament anatomically, defining this as a reconstruction that provides restoration of the ACL to its original dimensions, collagen orientation and insertion sites, in an attempt to replicate its anatomy. This might result in superior clinical results ${ }^{(2)}$.

The ideal way to reach the anteromedial (AM) and posterolateral (PL) femoral insertion sites of the ACL is through an accessory anteromedial (AAM) portal ${ }^{(3)}$. However, this route may produce femoral tunnels that are shorter than the transtibial route ${ }^{(4)}$, which may compromise the quality of the bone-graft interface ${ }^{(5)}$.

The positioning of the tunnels for placement of the graft is the most critical factor influencing the results from ACL reconstruction ${ }^{(6,7)}$. It is potentially influenced

1 - PhD in Orthopedics and Traumatology from the USP School of Medicine; Voluntary Attending Physician in the Orthopedics and Traumatology Service, Sorocaba School of Medical and Health Sciences, Pontificate Catholic University of São Paulo, Sorocaba, SP, Brazil.

2 - Former Resident in the Orthopedics and Traumatology Service, Sorocaba School of Medical and Health Sciences, Pontificate Catholic University of São Paulo, Sorocaba, SP, Brazil.

3 - Full Professor and Head of the Orthopedics and Traumatology Service, Sorocaba School of Medical and Health Sciences, Pontificate Catholic University of São Paulo, Sorocaba, SP, Brazil.

Work performed at Sorocaba School of Medical and Health Sciences, Pontificate Catholic University of São Paulo, Sorocaba, SP, Brazil.

Correspondence: Julio Cesar Gali, Av. Eugênio Salerno 387, 18035-430 Sorocaba, SP. E-mail: juliogali@globo.com

Work received for publication: March 17, 2011; accepted for publication: August 4, 2011.

The authors declare that there was no conflict of interest in conducting this work 
by, among other things, the angle of knee flexion at the time of drilling the tunnels, the locations of the portals and the anatomical variations between individuals ${ }^{(8)}$.

Our hypothesis here was that the greater the knee flexion was while drilling the femoral tunnels, the longer these tunnels would be. Thus, the objective of our study was to evaluate the effect that knee flexion might have on the length of the femoral tunnels.

\section{MATERIALS AND METHODS}

We measured and compared the lengths of anteromedial and posterolateral femoral tunnels constructed in 20 anatomical knee specimens. There were 10 right and 10 left knees, which were not in pairs and were of unknown sex and age. All the specimens presented intact joint cartilage and anterior and posterior cruciate ligaments.

The specimens had been fixed in $10 \%$ formol when fresh, and had been conserved in a mixture of $2.5 \%$ phenol, $2.5 \%$ formol and $1 \%$ sodium chloride. They were subsequently kept in liquid glycerin for 60 days, before dissection.

Using an open route, we removed the original AM and PL insertions of the ACL and marked out their centers using a bone pick.

The tunnels were drilled at the sites of these markers, using $2.5 \mathrm{~mm}$ bits, through the AAM portal. The anatomical specimens were flexed to construct the tunnels at $90^{\circ}, 110^{\circ}$ and $130^{\circ}$. The degree of flexion was determined using a goniometer that was aligned with the femoral and tibial diaphyses. The drilling was always done by two people: one maintaining the desired flexion, while the other did the drilling at the AM and PL femoral anatomical sites of the ACL (Figure 1).

We sought to keep the drill bit not more than 4 $\mathrm{mm}$ from the cartilage of the medial femoral condyle (MFC), always through the same entry point, in order to simulate intraoperative situations of arthroscopic reconstructions.

We did not use guides for the drilling. After removing the ACL, we marked out the femoral insertion points of the AM and PL bands and directed the bit laterally and slightly obliquely, towards the lateral femoral cortical bone (Figures 2 and 3). After completing this, we measured the tunnel lengths in millimeters, using a depth measuring device.

The statistical analysis was done using Friedman's analysis of variance and the Mann-Whitney test.

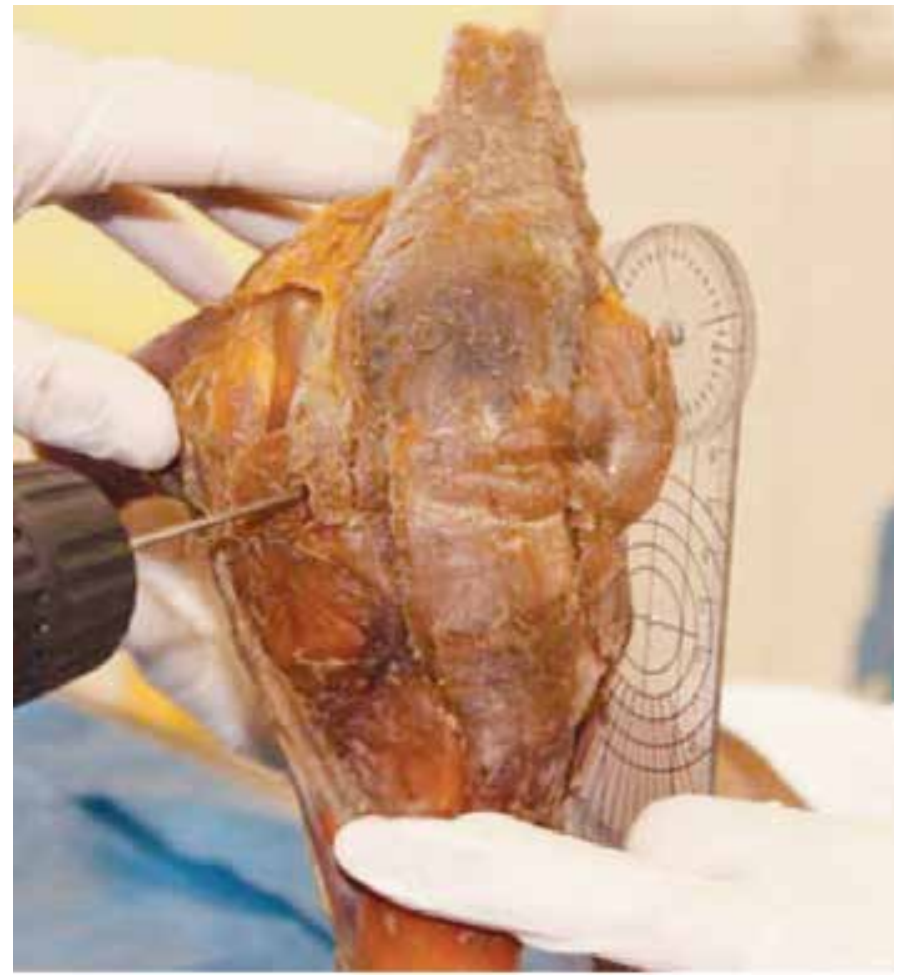

Figure 1 - Frontal view of an anatomical left-knee specimen with a drill bit inserted through the accessory anteromedial portal. The goniometer indicates the degree of knee flexion.

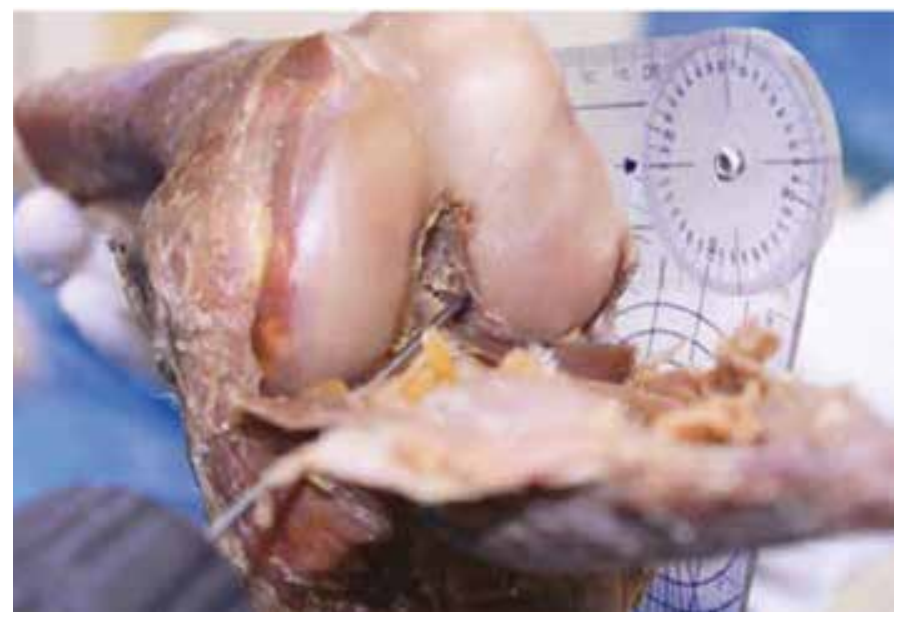

Figure 2 - Anatomical left-knee specimen with a drill bit inserted posterolaterally into the lateral femoral condyle.

\section{RESULTS}

The mean length of the AM femoral tunnels at $90^{\circ}$, $110^{\circ}$ and $130^{\circ}$ of flexion were, respectively, $33.7 \pm$ $3.7 \mathrm{~mm}, 37.4 \pm 2.9 \mathrm{~mm}$ and $38.8 \pm 3.3 \mathrm{~mm}$. For the PL femoral tunnels, the lengths were $32.1 \pm 4.2 \mathrm{~mm}$, $37.3 \pm 4.8 \mathrm{~mm}$ and $38.4 \pm 2.5 \mathrm{~mm}$ (Table 1).

In no case was there any violation of the posterior cortical bone of the AM femoral tunnel. Nor was there any damage to the lateral collateral ligament or to the popliteal tendon. 


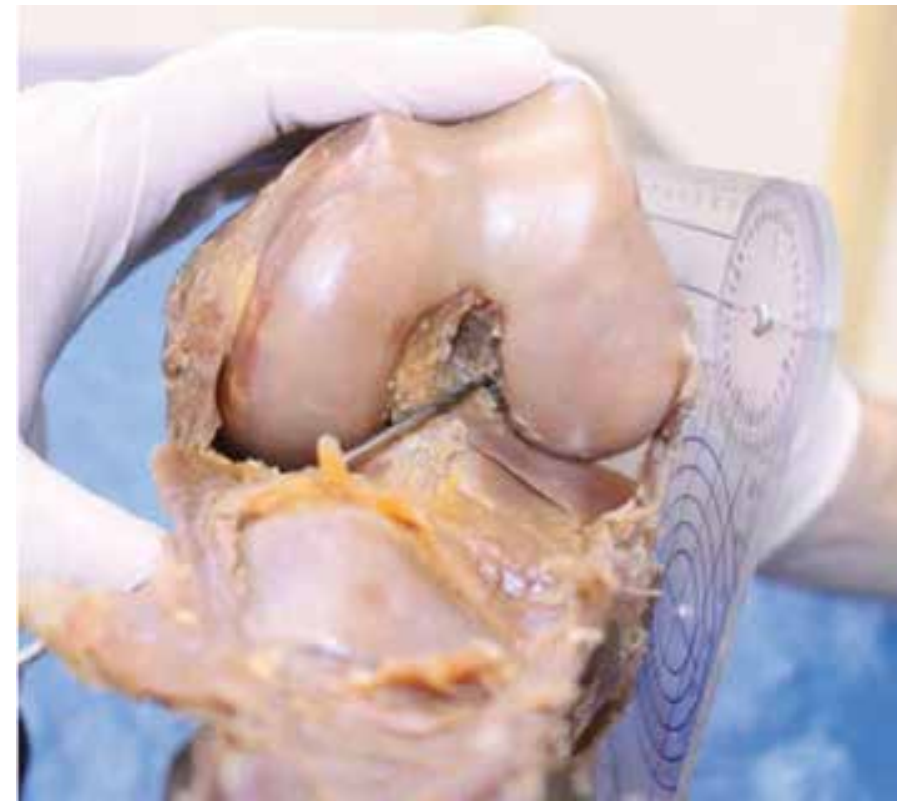

Figure 3 - Anatomical left-knee specimen with a drill bit inserted anteromedially into the lateral femoral condyle.

Table 1 - Lengths of the AM and PL femoral tunnels, in mm, at 90, 110 and 130 degrees of flexion.

\begin{tabular}{|c|c|c|c|c|c|c|}
\hline \multirow{2}{*}{ Knees } & \multicolumn{2}{|c|}{$90^{\circ}$} & \multicolumn{2}{|c|}{$110^{\circ}$} & \multicolumn{2}{|c|}{$130^{\circ}$} \\
\hline & AM & PL & AM & PL & AM & PL \\
\hline 1 & 30 & 31 & 39 & 36 & 42 & 39 \\
\hline 2 & 39 & 34 & 40 & 40 & 40 & 43 \\
\hline 3 & 33 & 32 & 36 & 36 & 37 & 37 \\
\hline 4 & 35 & 29 & 38 & 35 & 38 & 37 \\
\hline 5 & 34 & 30 & 36 & 33 & 37 & 34 \\
\hline 6 & 32 & 33 & 36 & 37 & 37 & 39 \\
\hline 7 & 32 & 30 & 34 & 36 & 36 & 37 \\
\hline 8 & 40 & 42 & 42 & 45 & 48 & 42 \\
\hline 9 & 31 & 30 & 36 & 37 & 36 & 38 \\
\hline 10 & 31 & 30 & 37 & 38 & 37 & 38 \\
\hline 11 & 38 & 30 & 40 & 34 & 42 & 40 \\
\hline 12 & 41 & 40 & 42 & 42 & 43 & 42 \\
\hline 13 & 30 & 32 & 34 & 33 & 39 & 36 \\
\hline 14 & 35 & 40 & 36 & 41 & 38 & 38 \\
\hline 15 & 32 & 30 & 35 & 32 & 36 & 34 \\
\hline 16 & 28 & 24 & 34 & 26 & 34 & 34 \\
\hline 17 & 30 & 32 & 37 & 38 & 38 & 39 \\
\hline 18 & 33 & 36 & 34 & 39 & 34 & 39 \\
\hline 19 & 38 & 32 & 39 & 38 & 40 & 38 \\
\hline 20 & 36 & 32 & 38 & 37 & 39 & 38 \\
\hline Mean & 33.9 & 32.45 & 37.15 & 36.65 & 38.55 & 38.1 \\
\hline SD & 3.72 & 4.24 & 2.53 & 4.05 & 3.31 & 2.51 \\
\hline
\end{tabular}

Abbreviations: $\mathrm{AM}=$ anteromedial; $\mathrm{PL}=$ posterolateral; $\mathrm{SD}=$ standard deviation.

Friedman's analysis of variance showed that the group with tunnels made at $90^{\circ}$ of flexion had significantly shorter tunnels than seen in the other groups, but it did not show any statistical difference between the groups measured with flexion of $110^{\circ}$ and $130^{\circ}(\mathrm{p}<0.05)$ (Table 2).
The Mann-Whitney test showed that the specimens came from different populations, i.e. the right and left knees could be summed to form a single heterogenous group ( $\leq 0.05)$ (Table 3$)$.

Table 2 - Length of the femoral tunnels, in $\mathrm{mm}$, drilled at 90,110 and 130 degrees of flexion.

\begin{tabular}{|c|c|}
\hline \multicolumn{2}{|c|}{$\begin{array}{c}\text { Friedman's analysis of variance } \\
\left(90^{\circ} \times 110^{\circ} \times 130^{\circ}\right)\end{array}$} \\
\hline AM & PL \\
\hline$x^{2} r=34.9$ & $x^{2} r=28.42$ \\
\hline$(\mathrm{P}<0.0001)$ & $(\mathrm{P}<0.0001)$ \\
\hline $110^{\circ}$ e $130^{\circ}>90^{\circ}$ & $110^{\circ}$ e $130^{\circ}>90^{\circ}$ \\
\hline
\end{tabular}

Abbreviations: $\mathrm{AM}=$ anteromedial; $\mathrm{PL}=$ posterolateral.

Table 3 - Mean lengths of the right and left tunnels.

\begin{tabular}{c|c|c|c}
\hline \multicolumn{4}{c}{ Mann-Whitney test (right vs. left) } \\
\hline \multirow{2}{*}{$\mathrm{AM}$} & $90^{\circ}$ & $110^{\circ}$ & $130^{\circ}$ \\
& $\mathrm{Z}=0.15$ & $\mathrm{Z}=0.52$ & $\mathrm{Z}=0.22$ \\
\hline $\mathrm{PL}$ & $\mathrm{Z}=0.68$ & $\mathrm{Z}=0.11$ & $\mathrm{Z}=0.18$ \\
\hline
\end{tabular}

Abbreviations: $\mathrm{AM}=$ anteromedial; $\mathrm{PL}=$ posterolateral.

\section{DISCUSSION}

The clinical results from surgical reconstruction of the ACL are influenced by a variety of factors. Among these is whether the tunnel location is perfect ${ }^{(6)}$ and the quantity of graft inside the tunnels ${ }^{(5)}$, which is ultimately determined by the length of the tunnel.

The final decision with regard to choosing the site for creating the tunnels depends on the surgeon's anatomical knowledge and arthroscopic view. The latter may be distorted by the positioning of the knee: thus, $90^{\circ}$ of flexion seems to be the angle that provides the best results regarding prevention of improper placement of the $\operatorname{graft}^{(8)}$.

In our study, the femoral tunnels were drilled through the AAM portal, given that the technique of constructing the femoral tunnel independently of the tibial tunnel makes it possible to construct femoral tunnels that are anatomically more correct ${ }^{(9)}$ and which function better ${ }^{(10)}$, in comparison with the technique of constructing femoral tunnels using the transtibial route.

However, the degree of knee flexion at the time of drilling may influence the tunnel length ${ }^{(11)}$. Technical reports have suggested that the drilling should be done at between $110^{\circ}$ and full flexion ${ }^{(12-16)}$.

Cha et $\mathrm{al}^{(12)}$ and Basdekis et $\mathrm{al}^{(13)}$ both recommended that the knee should be flexed at $110^{\circ}$ while the femoral drilling was performed. According to Zantop et $\mathrm{al}^{(14)}$, drilling at $110^{\circ}$ provided a safer distance from the subchondral bone and its cartilage. Bedi and 
Altchek ${ }^{(15)}$ advised flexion at $115^{\circ}$ before drilling, in order to increase the length of the tunnel upwards. According to Giron et $\mathrm{al}^{(16)}$, the femoral tunnel should be drilled through the AM portal at full flexion. Studies on cadavers, like ours, may more closely reflect the individual variation of knees $^{(14)}$. We sought to reproduce the surgical conditions for ACL reconstruction as closely as possible: we used the AAM portal to create the tunnels and we were able to directly view the AM and PL anatomical insertion sites of the ACL, in the lateral femoral condyle. Moreover, we took care not to injure the cartilage of the medial femoral condyle or the medial meniscus during the drilling.

We preferred to use $2.5 \mathrm{~mm}$ bits for creating the femoral tunnels, because provided that their origin in the lateral face of lateral femoral condyle was always the same, smooth wires might have create false paths.

As we increased the flexion of the anatomical specimens while drilling the tunnels, they began to be made more anteriorly, thus becoming more vertical in the frontal plane and longer (Figure 4). In a general manner, the length of femoral tunnels that are more lateral when drilled through the AM portal is shorter than in tunnels that are more anterior and medial, created via the transtibial route ${ }^{(17)}$.

In three published papers, greater knee flexion at the time of drilling produced longer femoral tunnels. Neven et $\mathrm{al}^{(17)}$ used cadavers and found measurements of between 32 and $44 \mathrm{~mm}$ (mean of $36.92 \mathrm{~mm}$ ) for the PL tunnel, which drilled through a low AM portal at $120^{\circ}$ of flexion, measured from inside to outside.

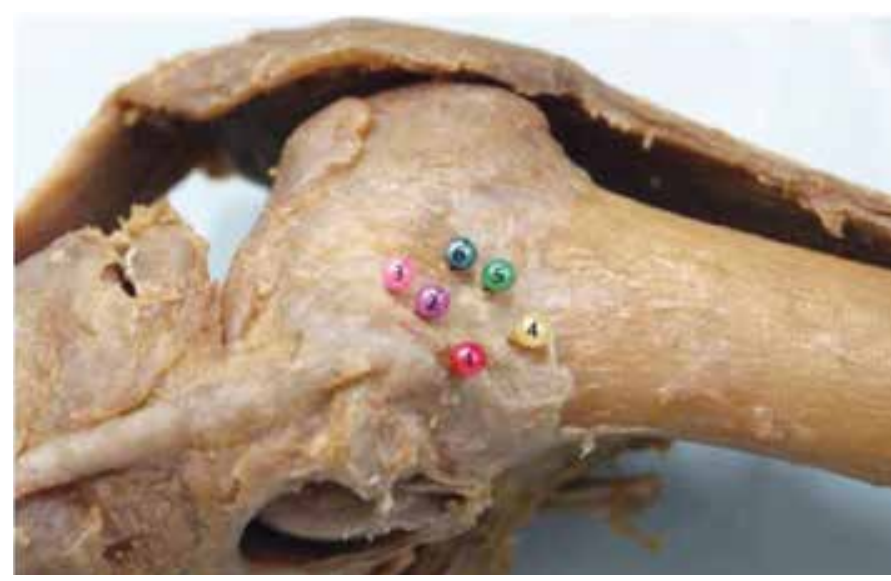

Figure 4 - Lateral view of an anatomical left-knee specimen. The markers 1,2 and 3 indicate the exit points in the lateral femoral condyle of the posterolateral tunnels drilled at 90,110 and 130 degrees of flexion, respectively. The markers 4,5 and 6 indicate the exit points in the lateral femoral condyle of the anteromedial tunnels drilled at 90,110 and 130 degrees of flexion, respectively. Increasing degree of flexion produced tunnels that were more vertical and anterior.
Basdekis et $\mathrm{al}^{(13)}$ evaluated the measurements of AM femoral tunnels drilled through an AM portal, in eight recent cadavers. The intraosseous measurements for the AM femoral tunnels, in mm, with the knees flexed at $90^{\circ}, 110^{\circ}, 130^{\circ}$ and maximum flexion were, respectively, $27.1 \pm 9.0,38.9 \pm 9.0,38.8 \pm 9.0$ and 39.2 \pm 4 . . However, both of these arthroscopic studies were done using the AL portal for viewing and the AM portal for drilling the tunnels, instead of placing the arthroscope in the AM portal, which would allow better viewing of the lateral intercondyle $e^{(18)}$.

In another study ${ }^{(19)}$, this time with nine recent cadavers, Basdekis et al measured the length of PL femoral tunnels drilled through the AM portal, with the anatomical specimens flexed at $90^{\circ}, 110^{\circ}$ and $130^{\circ}$, and found the following values in $\mathrm{mm}$, respectively: $33.2 \pm 2.6,35.4 \pm 4.0$ and $35.9 \pm 3.4$. They reported that the length did not vary significantly and that with $90^{\circ}$ of flexion, there was a risk of violating the posterior wall.

In our study, the drilling of the tunnels was done with direct viewing of the AM and PL femoral insertions of the ACL. Moreover, we used 20 knees that were not paired, i.e. a heterogenous group, which made our sample bigger than the three previous papers mentioned above.

On the other hand, in a study on nine pairs of knees from human cadavers in which the femoral tunnels were made by means of the AM route and were centered in the femoral insertions of the ACL, Bedi et $\mathrm{al}^{(20)}$ found the following measurements for the tunnels at $100^{\circ}, 110^{\circ}$ and $120^{\circ}$ of flexion, respectively: $30.9 \pm 2.6 \mathrm{~mm}, 25.7 \pm 5.4 \mathrm{~mm}$ and $21.3 \pm 4.8 \mathrm{~mm}$. The increase in flexion resulted in an increased risk of violating the posterior wall of the tunnel, and this occurred in $19.4 \%$ of the tunnels when they were made using the AM route.

Furthermore, they found a paradoxical reduction in tunnel length, which they believed could be explained by the use of a transtibial guide referenced to the posterior wall of the lateral femoral condyle. Theoretically, the greater the amount of graft material inside the bone tunnel was, the greater the amount of filling with collagen tissue would be, and the greater the chance of incorporation between the graft and the tunnel would be. Since lack of graft-bone integration could be a cause of failure of surgical reconstruction of the $\mathrm{ACL}^{(21)}$, the minimum quantity of graft 
material from soft tissues inside the bone tunnels for graft-bone union to occur without compromising the surgical results needs to be determined.

In an intra-articular model using sheep, Zantop et $\mathrm{al}^{(5)}$ performed reconstructions using grafts from the calcaneal tendon. They suggested that there was no negative correlation between grafts of length $15 \mathrm{~mm}$ in femoral tunnels and the resultant kinematic and structural properties. However, the minimum length of the graft inside the bone tunnels in humans has yet to be established.

Two points that could be considered to be weakness in our study can be indicated. The first is that in using anatomical specimens, the direction of the drilling of the tunnels could change and thus alter their lengths. To be able to avoid such occurrences, we entered the joint using the bit through the previously determined AAM portal and sought to keep it no more than $4 \mathrm{~mm}$ from the medial femoral condyle, in an attempt to simulate the surgical conditions. The second point is that we drilled three holes for each insertion of bands from the ACL, and this the drill could have taken a preestablished direction. To minimize this possibility, we did not use smooth wires to drill the tunnels, but preferred to use bits of $2.5 \mathrm{~mm}$ in diameter, in order to avoid creating false paths. The results from our study caused us to include some adjustments to our surgical practice: we promote slight hip flexion on the surgical table, so that the knee is flexed at $90^{\circ}$; we do not use a leg holder, in order to allow greater joint flexion; and we mark out the femoral insertion sites of the ACL with the knee flexed at $90^{\circ}$, to enable better viewing; and we drill with flexion of $110^{\circ}$.

\section{CONCLUSION}

It is possible to drill the AM and PL femoral tunnels with the knee flexed at $110^{\circ}$, in such a way that the minimum length is sufficient for bone-graft integration.

\section{ACKNOWLEDGEMENT}

We thank Prof. Dr. Neil Ferreira Novo for assistance with the statistical analysis in this study.

\section{REFERENCES}

1. Arnold MP, Kooloos J, van Kampen A. Single-incision technique misses the anatomical femoral anterior cruciate ligament insertion: a cadaver study. Knee Surg Sports Traumatol Arthrosc. 2001;9(4):194-9.

2. van Eck CF, Lesniak BP, Schreiber VM, Fu FH. Anatomic single- and double-bundle anterior cruciate ligament reconstruction flowchart. Arthroscopy. 2010;26(2):258-68.

3. Fu FH, Shen W, Starman JS, Okeke N, Irrgang JJ. Primary anatomic double-bundle anterior cruciate ligament reconstruction: a preliminary 2-year prospective study. Am J Sports Med. 2008;36(7):1263-74

4. Chang CB, Yoo JH, Chung BJ, Seong SC, Kim TK. Oblique femoral tunnel placement can increase risks of short femoral tunnel and cross-pin protrusion in anterior cruciate ligament reconstruction. Am J Sports Med. 2010;38(6):1237-45.

5. Zantop T, Ferretti M, Bell KM, Brucker PU, Gilbertson L, Fu FH. Effect of tunnel-graft length on the biomechanics of anterior cruciate ligament-reconstructed knees: intra-articular study in a goat model. Am J Sports Med. 2008;36(11):2158-66.

6. Aglietti P, Giron F, Losco M, Cuomo P, Ciardullo A, Mondanelli N. Comparison between single-and double-bundle anterior cruciate ligament reconstruction: a prospective, randomized, single-blinded clinical trial. Am J Sports Med. 2010;38(1):25-34.

7. Khalfayan EE, Sharkey PF, Alexander AH, Bruckner JD, Bynum EB. The relationship between tunnel placement and clinical results after anterior cruciate ligament reconstruction. Am J Sports Med. 1996;24(3):335-41.

8. Hoshino Y, Nagamune K, Yagi M, Araki D, Nishimoto K, Kubo S, et al. The effect of intra-operative knee flexion angle on determination of graft location in the anatomic double-bundle anterior cruciate ligament reconstruction. Knee Surg Sports Traumatol Arthrosc. 2009;17(9):1052-60.

9. Abebe ES, Moorman CT 3rd, Dziedzic TS, Spritzer CE, Cothran RL, Taylor DC, et al. Femoral tunnel placement during anterior cruciate ligament reconstruction: an in vivo imaging analysis comparing transtibial and 2-incision tibial tunnel-independent techniques. Am J Sports Med. 2009;37(10):1904-11.

10. Steiner ME, Battaglia TC, Heming JF, Rand JD, Festa A, Baria M. Independent drilling outperforms conventional transtibial drilling in anterior cruciate ligament reconstruction. Am J Sports Med. 2009;37(10):1912-9.

11. Golish SR, Baumfeld JA, Schoderbek RJ, Miller MD. The effect of femoral tunnel starting position on tunnel length in anterior cruciate ligament reconstruction: a cadaveric study. Arthroscopy. 2007;23(11):1187-92.

12. Cha PS, Brucker PU, West RV, Zelle BA, Yagi M, Kurosaka M, et al. Arthroscopic double-bundle anterior cruciate ligament reconstruction: an anatomic approach. Arthroscopy. 2005;21(10):1275.

13. Basdekis $G$, Abisafi $C$, Christel P. Influence of knee flexion angle on femoral tunnel characteristics when drilled through the anteromedial portal during anterior cruciate ligament reconstruction. Arthroscopy. 2008;24(4):459-64.

14. Zantop T, Haase AK, Fu FH, Petersen W. Potential risk of cartilage damage in double bundle ACL reconstruction: impact of knee flexion angle and portal location on the femoral PL bundle tunnel. Arch Orthop Trauma Surg. 2008;128(5):509-13.

15. Bedi A, Altchek DW. The "footprint" anterior cruciate ligament technique: an anatomic approach to anterior cruciate ligament reconstruction. Arthroscopy. 2009;25(10):1128-38.

16. Giron F, Buzzi R, Aglietti P. Femoral tunnel position in anterior cruciate ligament reconstruction using three techniques. A cadaver study. Arthroscopy. 1999;15(7):750-6.

17. Neven E, D'Hooghe $P$, Bellemans J. Double-bundle anterior cruciate ligament reconstruction: a cadaveric study on the posterolateral tunnel position and safety of the lateral structures. Arthroscopy. 2008;24(4):436-40

18. Fu FH, Shen W, Starman JS, Okeke N, Irrgang JJ. Primary anatomic double-bundle anterior cruciate ligament reconstruction: a preliminary 2-year prospective study. Am J Sports Med. 2008;36(7):1263-74.

19. Basdekis G, Abisafi C, Christel P. Effect of knee flexion angle on length and orientation of posterolateral femoral tunnel drilled through anteromedial portal during anatomic double-bundle anterior cruciate ligament reconstruction. Arthroscopy. 2009;25(10):1108-14.

20. Bedi A, Raphael B, Maderazo A, Pavlov H, Williams RJ 3rd. Transtibial versus anteromedial portal drilling for anterior cruciate ligament reconstruction: a cadaveric study of femoral tunnel length and obliquity. Arthroscopy. 2010;26(3):342-50.

21. Carson EW, Anisko EM, Restrepo C, Panariello RA, O'Brien SJ, Warren RF. Revision anterior cruciate ligament reconstruction: etiology of failures and clinical results. J Knee Surg. 2004;17(3):127-32. 